Itinéraires Itinéraires

Littérature, textes, cultures

2020-2 | 2020

Discours animal. Langages, interactions, représentations

\title{
Chiens sentients : du langage émotif au discours critique
}

Quelques exemples de personnages de chiens dans des récits littéraires et cinématographiques

Sentient Dogs: From Emotional Language to Critical Discourse. Some Examples of Dog Characters in Literary and Cinematographic Narratives

\section{Maria do Rosário Girão Ribeiro Dos Santos}

\section{OpenEdition}

Journals

\section{Édition électronique}

URL : https://journals.openedition.org/itineraires/8413

DOI : $10.4000 /$ itineraires.8413

ISSN : 2427-920X

Éditeur

Pléiade

\section{Référence électronique}

Maria do Rosário Girão Ribeiro Dos Santos, « Chiens sentients : du langage émotif au discours critique », Itinéraires [En ligne], 2020-2 | 2020, mis en ligne le 22 décembre 2020, consulté le 25 novembre 2021. URL : http://journals.openedition.org/itineraires/8413 ; DOI : https://doi.org/10.4000/ itineraires.8413

Ce document a été généré automatiquement le 25 novembre 2021.

\section{c) (i)}

Itinéraires est mis à disposition selon les termes de la licence Creative Commons Attribution - Pas d'Utilisation Commerciale - Pas de Modification 4.0 International. 


\section{Chiens sentients : du langage émotif au discours critique}

Quelques exemples de personnages de chiens dans des récits littéraires et cinématographiques

Sentient Dogs: From Emotional Language to Critical Discourse. Some Examples of Dog Characters in Literary and Cinematographic Narratives

Maria do Rosário Girão Ribeiro Dos Santos

\section{Introduction}

1 Depuis René Descartes ${ }^{1}$ et Emmanuel Kant ${ }^{2}$, qui prônent une différence métaphysique entre l'être humain et l'être non humain, ainsi qu'une vision mécaniciste de l'animal, envisagé comme automate, dépourvu de raison et de conscience, et Darwin ${ }^{3}$, pour lequel la susdite différence est de degré et non de nature, la question animale et celle de la spécificité humaine ont beaucoup évolué. Les nouvelles découvertes scientifiques autour des débats sur le spécisme ${ }^{4}$ et l'antispécisme, l'anthropocentrisme et le zoocentrisme ont révolutionné les études sur l'être vivant et débouché sur les « animal studies » et les « human-animal studies ${ }^{5} »$.

2 L'animal, figure de l'Antiquité, de la modernité ${ }^{6}$ et de la surmodernité ${ }^{7}$, se trouve en effet au carrefour de plusieurs aires disciplinaires, de la biologie à l'anthropologie, de la génétique à la cybernétique, de la philosophie à la psychologie biologique, de l'écologie à l'éthique appliquée ${ }^{8}$, des études littéraires à la bande dessinée et au cinéma, de l'éthologie cognitive à la zoopoétique. Cette trajectoire est illustrée, par exemple, par Michel Foucault, qui se penche sur l'animalité et la folie (Histoire de la folie, 1961) ; par Donna Haraway qui publie The Companion Species Manifesto: Dogs, People and the Significant Otherness (2003) ; par Peter Singer qui, dans La Libération animale (1975), défend la thèse que les êtres sensibles doivent être considérés comme moralement égaux et s'insurge contre la discrimination de quelques êtres qui appartiennent à une espèce biologique non humaine; par Élisabeth de Fontenay, qui critique, dans Le Silence des bêtes : la 
philosophie à l'épreuve de l'animalité (1998), l'ethnocentrisme humaniste et remet en cause la différence entre l'homme et l'animal; ou encore par Corine Pelluchon, selon laquelle, dans Éléments pour une éthique de la vulnérabilité (2011), l'écologie et la cause animale vont de pair dans un nouvel humanisme.

3 Jacques Derrida, créateur du terme animot, réfute quant à lui l'inclusion de tous les êtres vivants non humains dans la catégorie commune et générale de l'animal et soutient le besoin d'accéder à une forme de pensée susceptible de contourner l'absence du nom ou du mot (et non de restituer le mot aux animaux) (2002: 89). Il dénonce ainsi le langage comme instrument culturel de discrimination et d'exploration de l'Autre (humain ou non humain). Pour Anne Simon (2017a: 83), la zoopoétique, en reliant "puissance inventive [...] du langage créatif et expressivité primordiale du vivant", "prend au sérieux la diversité des actes et des comportements qui engagent les bêtes [...] dans des formes multiples d'expressivité » (84), devenant une zoopoéthique « quand elle se demande ce que serait le monde sans le cinquième jour, où apparaissent les Aquatiques et les Ailés [...]» (93).

Nous nous proposons d'étudier dans cet article quelques exemples de récits fictionnels dans lesquels les animaux sont dotés de capacités de sentience, et qui permettent d'explorer la manière dont cette reconnaissance, sérieuse ou humoristique, de compétences animales, enrichit la relation homme/animal. Notre corpus est constitué de six récits qui mettent en scène des chiens. Les trois premiers sont révélateurs de la relation particulière qui unit le chien et son maître : Flush. A Biography (Virginia Woolf, 1933), Timbuktu (Paul Auster, 1999), A través de mis pequeños ojos (Emilio Ortiz, 2016). Les trois suivants portent plus spécifiquement sur la thématique du retour et de l'attente, qui marquent un degré important de sentience engageant mémoire et émotion : Cão como nós (Manuel Alegre) et deux films intitulés Hachi: A Dog's Tale (2009) et A Dogs's Purpose (2017). Nos critères de sélection ont été les suivants : le degré de sentience ${ }^{9}$ des chiens; la gradation croissante en ce qui concerne la représentativité du narrateur (humain et non humain), ainsi que la pertinence du point de vue (humain et animal); l'unité, au niveau fonctionnel et émotionnel, du protagoniste humain et canin ; enfin, l'égalité du chien sentient et de l'homme conscient de sa sentience.

\section{La sentience animale}

5 Il convient tout d'abord de définir le concept de sentience : être sentient signifie être conscient, avoir la capacité d'être affecté, d'une façon positive ou négative, par des expériences distinctes, en ayant des perceptions conscientes du milieu environnant et en réagissant à un stimulus donné et à des sensations, physiques et psychologiques, telles que le froid, le confort, la douleur et la souffrance ${ }^{10}$. Pour Donald M. Broom, auteur de Sentience and Animal Welfare (2014) et du rapport européen Le bien-être animal dans l'Union européenne (2017), l'être sentient ${ }^{11}$ est en outre capable d'évaluer ses actions, ainsi que les actions/réactions des autres par rapport aux siennes; de se rappeler ses actions, de se souvenir de leurs conséquences et d'en évaluer les risques et les bénéfices; de ressentir des sentiments et des émotions et d'avoir un degré variable de conscience. La sentience est donc plus qu'une simple conscience : elle implique des capacités d'évaluation, de mémoire et de ressenti.

6 Dans ce contexte, et à propos de la conscience, António Damásio distingue trois niveaux sous-jacents au moi : si le proto et le nucléo, fondés sur les sensations, concernent toutes 
les espèces, le moi autobiographique est construit sur des souvenirs (passés) et des projections (futures), conduisant à une mémoire amplifiée et, par conséquent, à la logique, à la créativité, au langage et à l'émotion. Bien qu'émotion et sentiment soient dans la plupart des cas employés indistinctement, il faut toutefois essayer de saisir les particularités définitoires de l'une et de l'autre, du point de vue de leur durée/ intensité, de leur manifestation, de leur nature et en proposer une typologie à l'intérieur de chaque catégorie. Les émotions, jouant un rôle de régulation homéostatique, sont transitoires ou contingentes. Malgré leur intensité, elles se manifestent extérieurement et relèvent de la neuro-psychophysiologie (Damásio 2000a: 71-81). Les sentiments priment par leur durabilité, intériorité et spiritualité, s'éloignant du théâtre du corps. Damásio propose une typologie constituée de trois types d'émotions et de deux variétés de sentiments. D'une part, les émotions primaires ou universelles (comme la surprise et le dégoût), les émotions secondaires ou sociales (dont la honte, la jalousie, la culpabilité et l'orgueil) et les émotions de fond (telles que le bien-être et le malaise, le calme et la tension). D'autre part, les sentiments basés non seulement sur les émotions, mais aussi sur leurs variantes; l'euphorie ou l'extase, apparaissent comme des variantes du bonheur, la mélancolie ou l'anxiété comme des variantes de la tristesse et la timidité ou la panique comme des variantes de la peur. Pour éviter le risque d'une généralisation peu rigoureuse, on pourrait même avancer avec António Damásio (1998: 153 et suiv.) que toutes les émotions donnent lieu à des sentiments, quoiqu'il y ait des sentiments, comme c'est le cas des sentiments de fond, qui ne proviennent pas des émotions. D'ailleurs, selon ce neurologue, il existe un état d'émotion, qui peut être déclenché et exécuté d'une façon inconsciente, un état de sentiment, qui peut être représenté inconsciemment, et un état de sentiment qui devient conscient, tout en étant reconnu par l'organisme qui ressent de l'émotion et du sentiment (2000b : 75) ${ }^{12}$. À l'instar des hommes, les animaux sont sentients : d'ailleurs, cette souffrance qu'ils partagent avec l'être humain s'avère l'un des fondements de la considération éthique que l'on éprouve à leur égard (Bentham 1789).

Parmi les animaux ${ }^{13}$ doués de sentience, les chiens occupent une place particulière. En raison d'abord de l'évolution conjointe de l'homme et des canidés; ensuite de leur héritage symbolique et mythique. Liée à la "trinité » terre, eau et lune, la fonction primordiale du chien est en effet celle de "psychopompe", de "guide de l'homme durant la nuit de la mort après avoir été son compagnon durant le jour de la vie» (Chevalier et Gheerbrant $1969: 239)^{14}$. Le chien conquiert ainsi au fil des siècles le statut de «meilleur ami de l'homme ${ }^{15}$ » et, partageant avec lui l'oikos, joue son rôle ancestral de guide, devient une figure familière et une figure familiale, jusqu'à devenir un membre de la famille assimilé civilement à un enfant pour lequel ses parents adoptifs peuvent éventuellement se disputer ${ }^{16}$.

\section{Chiens errants}

Dans Flush. A Biography (publié en 1933) ${ }^{17}$, Virginia Woolf, lectrice de Proust et de Joyce, retrace le parcours existentiel du chien Flush, le cocker spaniel d'Elizabeth Browning.

Si l'écrivaine moderniste, fille d'un victorien éminent, manifeste sa rupture avec la société victorienne, dénoncée comme ridicule, elle lui rend hommage par une visionécriture dynamique de la tradition, susceptible d'être illustrée par la parodie darwinienne (La Traversée des apparences) et par le pastiche ruskinien (Orlando). En ce 
qui concerne le récit (Flush), elle s'inspire des lettres et des poèmes que Miss Barrett a consacrés à son chien, en alternant la fiction et l'essai, en retraçant une biographie imaginaire, en réinventant en somme le victorianisme à travers la coexistence harmonieuse de la convention et de l'innovation. Dans Flush, ainsi que dans ses autres récits, elle renonce à la focalisation omnisciente du narrateur au profit de la voix intérieure des personnages (en particulier, de Miss Barrett et de Flush), de leur expérience de la vie et de leurs émotions dans un déroulé narratif, « qui n'est plus réglé comme un métronome, mais scandé par des moments d'intensité » (Reynier 2012: 64-65). Dotée d'une "curiosité prodigieuse", elle se "transforme, pour quelques heures, en femme d'intérieur", la vie quotidienne devenant, de la sorte, "héroïque, scintillante, aventureuse » (Citati $2003: 104-105)$.

Elle écrit la biographie ${ }^{18}$ d'un chien aristocrate, ce qui annonce déjà un changement de paradigme par rapport aux biographies traditionnelles. Ce récit de vie est en outre enchâssé, comme une " mise en abyme ", dans un autre (celui d'Elizabeth et de Robert) à « nuances spéculaires » (Dallenbach 1977), étant donné qu'au fur et à mesure qu'elle narre la trajectoire existentielle de Flush, elle relate, également, le parcours de vie du couple Barrett-Browning. De même, la prolifération des points de vue, qui bouleverse les fondements et renouvelle les techniques du roman, fait avancer l'intrigue, moins de l'extérieur que du côté du flux de la conscience. L'un des exemples les plus réussis de cette focalisation plurielle, mobile ou changeante, est illustré par la panoplie d'émotions ressenties par Flush lors de la naissance du bébé du couple Browning : son anxiété nerveuse qu'un pressentiment déclenche; sa rage et sa jalousie devant cet " animal vivant ${ }^{19}$ ", cette chose horrible qui "s'agitait et miaulait à ses côtés » (Flush, $2016: 83)^{20}$; son aversion à l'égard d'une " présence repoussante ${ }^{21}$ ", qui l'intrigue et le tourmente; sa profonde mélancolie visible dans son indifférence vis-à-vis des attentions redoublées que la famille (sa famille) lui prodigue; son affection naissante et croissante pour ce bonhomme faible et pleurnicheur, qui ne tarde pas à le métamorphoser en cheval ${ }^{22}$; enfin, l'amitié réciproque entre le bébé et le chien ${ }^{23}$, qui partagent les mêmes goûts pour le paysage environnant (insipide pour les deux, mais objet d'extase pour Mme Browning) et en ce qui concerne l'incapacité littéraire. En outre, Elizabeth et Flush présentent, au niveau de la prosopographie, des ressemblances étonnantes: les boucles/poils qui encadrent le visage/le museau, les grands yeux brillants, la même bouche, grande et souriante. Profondément sensible aux émotions humaines, quoiqu'il ait du mal à comprendre celles d'Elizabeth, Flush n'a pas besoin d'interagir avec des mots : ceux-ci, questionne le double narrateur, réussissent-ils à tout exprimer? Ne détruisent-ils pas les symboles qui sont au-delà des mots euxmêmes? (23-24). D'une part, le fait de ne pas communiquer avec des mots, ce qui « [...] était, sans aucun doute, responsable de beaucoup de malentendus ${ }^{24} »(23)$; d'autre part, cette impuissance langagière, comblée par le protolangage qu'est l'émotion, deviennent un tremplin pour une "intimité particulière ${ }^{25}$ » (ibid.). En ce qui concerne les coordonnées spatiales et visuelles ${ }^{26}$, il faut mettre en relief l'Angleterre et l'Italie, le Londres victorien (dont la fausse moralité n'est partagée ni par la biographe-narratrice ni par le narrateur canin) - traversé par la richesse et la pauvreté - et les villes de Pise et de Florence. Les émotions ressenties à l'égard des lieux par la poétesse Elizabeth et le chien Flush façonnent, en termes imagologiques, l'italophilie et l'anglophobie. Tout d'abord, la somnolence londonienne contraste avec le bruit assourdissant à Pise : «[...] il y avait un bruit infernal de cris, de clameurs, un claquement de fouets, des sons métalliques et un retentissement de cloches $^{27} »$ (73). Cette image d'un espace 
fourmillant et animé, d'une Italie libre, heureuse et ensoleillée, est constamment louée par l'épouse de Robert Browning, qui «se plaignait de sa pauvre Angleterre, humide, ennuyeuse, sans soleil, sans joie, coûteuse et conventionnelle ${ }^{28}$ » (75-76). Flush, quant à lui, s'aperçoit vite des différences inhérentes aux «castes» canines, anglaises et italiennes: tandis qu'à Londres la race ou le pedigree sont les garants de la reconnaissance sociale, à Pise il devient impossible de distinguer un "pug dog, retriever, bulldog, mastiff, collie», ce qui le transforme en un prince exilé, seul aristocrate dans une multitude de canailles, « le seul cocker spaniel de pure race ${ }^{29}$ » (74). En ce qui concerne les puces qui l'attaquent, celles de Florence sont «rouges et viriles $^{30}$ ", c'est-à-dire difficiles à tuer, à l'inverse de celles de Reading qui s'écrasent facilement (89).

11 Il convient de souligner, à propos de Flush. A Biography, l'importance de la complicité entre Virginia Woolf et Elisabeth Barrett (deux femmes intellectuelles et écrivaines), à travers la présence inoubliable de Flush, ainsi que la profusion de points de vue : Flush et Elizabeth, par leurs points de vue alternés, forment comme un narrateur pluriel qui, doué d'un indéniable et exceptionnel "sens de l'humour ${ }^{31}$ ", rend manifestes les relations ambiguës entre le moi et l'autre, envisagés comme moralement égaux, selon Peter Singer. Moyennant la convivialité, l'émotion et l'amitié, l'entente est parfaite, réciproque, les barrières linguistiques étant surmontées au profit des gestes symboliques, dans un récit caractérisé par la fluidité, l'évanescence et la modulation.

12 Tandis que Flush est un chien aristocrate, Mr. Bones, le chien protagoniste de Timbuktu ${ }^{32}$ de Paul Auster (1999), est un vagabond philosophe, qui vit dans un contexte sociohistorique et psychofamilial différent. Mr. Bones, Canis familiaris (pour ne pas dire mâtin), parcourt l'Amérique avec William, dont il est le compagnon fidèle ou le « kemo sabe $^{33}$ ». Arrivé à Baltimore, Willy, poète errant et vagabond excentrique, qui pressent sa mort imminente, tente de rencontrer son ex-professeure, afin de lui rendre ses cahiers de poèmes et de lui confier son ami à quatre pattes. Quoique Mr. Bones ne soit pas un chien de race, à l'image du snob Flush, il lui est supérieur par son intelligence et par son «don » linguistique, qui lui est attribué par l'humour du narrateur: outre la connaissance de ce protolangage qu'est l'expression de l'émotion, il comprend tout ce que son ami humain lui dit. D'ailleurs, l'« ingloosh» (langue adultérée par l'influence de celle des immigrants) est sa deuxième langue, la première étant la langue canine. S'il maitrise la syntaxe et la grammaire, la prononciation lui pose en revanche quelques problèmes : la configuration de son museau (ainsi que celle de ses dents et de sa langue) semble être coupable de son discours décousu, malgré ses efforts herculéens pour imiter le langage humain. Cet obstacle, vite surmonté, n'empêche pas une interaction/ communication harmonieuse(s) entre le chien non humain et l'homme non chien: Willy, qui l'écoute attentivement, l'envisage comme un "un ange prisonnier dans un corps de chien ${ }^{34}$ "(Timbuktu, 2008: 35) ou, plutôt, grâce à l'anagramme, comme "God»: "Quelque chose aurait-il pu être plus évident? Il suffisait de permuter les lettres du mot dog et qu'est-ce qu'on obtenait ?35 »(36). Quant à Mr. Bones, il est d'avis que Willy «était un homme avec un cœur de chien ${ }^{36}$ » (30). Les affinités entre Willy et Mr. Bones (ou Bonesy, son diminutif) les unissent pour la vie : la nourriture, le sexe et la sociabilité, évoquée par la lecture des journaux (qui renseignent l'homme sur ce que ses semblables sont en train de faire) et dans le flairage des arbres et des poteaux d'éclairage (qui informent le chien sur la population canine et locale). Si différence il y a, elle ne tient qu'à la supériorité des récepteurs olfactifs des chiens, façonnant leur «paradis nasal» (40): "Un chien avait, environ, deux cents vingt millions de 
récepteurs olfactifs, tandis qu'un être humain n'en avait que cinq millions [...] $]^{37}$ (39). C'est, d'ailleurs, cette « suprématie » canine (dont le flair est l'emblème) qui force Willy à se questionner sur le temps d'une symphonie, ainsi que sur la panoplie d'odeurs qu'elle est susceptible de contenir, voire sur le projet inédit de l'invention d'une "Symphony of Smells» (35). À l'origine de cette recherche novatrice, parsemée d'humour, se trouve l'insuffisance des connaissances du poète vagabond, corroborée par l'humour du narrateur, à l'égard des poses canines, qu'il est urgent de connaître, maîtriser, assimiler :

Il y avait tant de choses à absorber, tant d'évidences à assimiler, à déchiffrer, à comprendre, que Willy ne savait même pas par où commencer. Le remuement de la queue en opposition à la queue basse. Les oreilles hérissées s'opposant aux oreilles moles. Le ventre vers le haut, les courses en cercles [...] et une centaine d'autres détails ${ }^{38}$. (37)

13 À Willy de conclure que ces efforts sisyphéens et prométhéens s'apparentent à " apprendre à parler un nouveau langage ${ }^{39}$ » (ibid.). D'une part, Sir Osso, autre surnom du chien (61), s'entête à parler, malgré les obstacles biologiques, la langue de Willy, tout à fait convaincu que son chien a une âme (38); d'autre part, Willy, prêt à surmonter n'importe quel obstacle, s'ingénie à comprendre celle de Bonesy, tous les deux effaçant et détruisant le dualisme conventionnel. De péripétie en péripétie, Mr. Bones, après le décès de Willy, rêve de la légendaire Timbuktu - «La carte de Timbuktu commence où la carte de ce monde se termine ${ }^{40} »(49)$-, mentionnée à plusieurs reprises par son ami. Il s'agit, d'abord, d'une fascination d'ordre phonétique qu'exerce sur lui la combinaison des phonèmes : «Tim-buk-tu. [...] La combinaison incisive des voyelles et des consonnes avait le don de le toucher, dans la partie la plus profonde de son âme [...] $]^{41}$ » (ibid.). C'est ensuite la fascination d'ordre géographique qu'une localisation quasi irréelle suscite, en activant l'imaginaire ${ }^{42} \mathrm{du}$ chien :

Ce lieu s'appelait Timbuktu, et autant que Mr. Bones avait pu comprendre, il était situé quelque part au milieu d'un désert, loin de New York ou Baltimore, loin de la Pologne ou de quelque autre ville qu'ils avaient visitée au cours de leurs voyages ${ }^{43}$.

C'est encore la fascination déclenchée par l'utopie et l'uchronie, et par le sortilège de l'endroit, l'esprit du lieu - décrit par Willy comme une «oasis d'esprits» (49) -, où il espère rencontrer et garder à jamais « l'esprit » de son ami. C'est enfin la fascination antispéciste, tendant vers l'égalité entre l'animal-humain et l'animal non humain: «[...] à Timbuktu les chiens seraient capables de parler le langage humain et de traiter d'égal à égal avec l'homme ${ }^{44} »(50)$. Et c'est vers cet horizon perdu, cette utopie, aux antipodes de la dystopie américaine (dont le paradigme paraît être Baltimore), que Mr. Bones, vieux, malade et fou, s'achemine, en faisant «de l'auto-stop» sur l'autoroute, anxieux de retrouver Willy : « Avec un peu de chance, il aurait pu retrouver Willy avant la fin de la journée ${ }^{45}$ » (186). À l'instar du narrateur-auteur, qui pénètre maintes fois dans le récit ${ }^{46}$ et qui affirme, de façon péremptoire, l'égalité entre un homme et un chien ("qui dit un type dit un chien»), nous assistons, en tant que lecteurs, à cette fusion entre le chien et l'homme, à cette unité entre deux espèces et à ce respect réciproque qui, selon Derrida, dénoncent le langage comme étant un facteur de discrimination et d'exploration de l'autre.

Cette unité, fonctionnelle et émotionnelle, est évidente dans le roman de Emilio Ortiz, daté de 2016 et intitulé $A$ través de mis pequeños ojos. Devenu un best-seller ${ }^{47}$, l'ouvrage met en scène un golden retriever, prenant en charge l'énonciation, non seulement en tant que narrateur-autobiographe ${ }^{48}$, mais aussi en tant que biographe de l'aveugle 
Mario. Son statut de chien guide permet de mettre en relief la "pluralité des moyens stylistiques, linguistiques et narratifs» dont se sert l'écrivain afin de restituer la "diversité des comportements " et la «complexité des interactions homme-animal » (Simon 2017b : 16), traduites, en l'occurrence, par le discours du narrateur canin, le monologue du chien guide et la critique de l'homme et de la société, évidente dans le parallélisme établi entre les humains et les non-humains. Ensemble, Mario et Cross accomplissent pendant treize ans un parcours existentiel qui commence à l'école de formation pour les aveugles et se termine par le décès du protagoniste canin. Ce double parcours, qui devient unique, déterminé par une pédagogie dictée par l'apprentissage mutuel, est parsemé d'émotions, étant donné que, d'après le narrateur canin doué d'humour, les chiens guides sont semblables aux humains pour ce qui est de l'aspect sentimental: «[...] Nous, les chiens-guides, nous ressemblons à nos maittres en des choses très concrètes, $\mathrm{du}$ moins en ce qui concerne les questions sentimentales ${ }^{49} \gg$ (Ortiz $\left.2016: 74\right)$.

Parallèlement aux hommes qui suivent et subissent des épreuves à l'école, au lycée, à l'université et au travail, les chiens vivent aussi des étapes existentielles, des tranches de vie qui les conduisent à une véritable initiation. Cependant, qui dépend le plus de qui ? Le chien de son maître? Ou le maître de son chien?, s'interroge le chien narrateur.

Outre les liens professionnels et émotionnels qui s'établissent entre eux, le roman apparaît comme un pamphlet écologique et comme une critique de l'homme, à peine atténuée par l'humour: "Pourquoi, au lieu de recourir à nous, ils ne tentent pas de faire ce que nous faisons ? De vivre comme nous vivons ${ }^{50}$ ?» (114).

17 La réponse est simple : d'après Cross, l'homme n'a pas besoin d'imiter les chiens, mais tout simplement de remonter à ses origines, aux temps immémoriaux où il était encore libre et heureux. Une réminiscence de Rousseau, surtout du Discours sur l'origine de l'inégalité entre les hommes? Nonobstant, un jour arrive où il prend la décision de tout contrôler, d'usurper le premier rang de la hiérarchie naturelle en finissant par devenir esclave de lui-même. Entre-temps, il a modifié la nature (afin de garantir sa survie), en créant les villes, en tuant - «des blousons faits avec [la peau] des animaux morts ${ }^{11} »(108)$ - les petits animaux, en détruisant les fruits et les légumes frais et en supprimant les étangs où l'on pourrait boire. Un écho de Thoreau ${ }^{52}$ ? À vrai dire, Henry David Thoreau, dans une conférence donnée au «Concord Lyceum» le 23 avril 1851, a annoncé son projet de « dire quelques mots au nom de la Nature, de la liberté absolue et de l'état sauvage, en contraste avec la liberté et la culture [...] civilisées, ayant comme objectif de voir dans l'homme un habitant, une parcelle de la nature plutôt qu'un membre de la société » (Thoreau [1854] 2014 : 15). De même, dans Walden ou la vie dans les bois, l'écrivain nord-américain, source d'inspiration de plusieurs générations d'Américains en ce qui concerne la valorisation de l'état sauvage et des droits / devoirs civiques, a affirmé que la seule "vraie Amérique» est celle où l'on peut vivre librement, sans esclavage, guerre et d'autres dépenses superflues (Thoreau [1854] 2014 : $229,230)^{53}$. Tout en poursuivant son réquisitoire - selon lequel l'écologie et la cause animale vont de pair - qui, exempt de férocité, relève d'un ethos peu agressif, mais incisif, Cross n'hésite pas à énumérer d'autres divergences mineures: tandis que les chiens deviennent tristes quand ils sont seuls, cette tristesse cédant la place à la joie, l'être humain, après la résolution de ses problèmes, continue à patauger dans la souffrance. Contrairement à cette espèce bizarre ${ }^{54}$ qui ne réussit pas à se séparer de ses 
enfants, les chiens libèrent les leurs, une fois leur obligation accomplie. D'autres différences (qui façonnent le niveau idéologique du narrateur, ainsi que son indéniable humour) sont graduellement distillées, relevant d'une simple constatation des faits par un regard philosophe-critique à effets comiques: pourquoi les hommes mangent-ils cinq fois par jour et les chiens une seule fois ${ }^{55}$ ? (34). Comment expliquer qu'une salle de bains soit à l'intérieur et non pas en plein air ${ }^{56}$ ? (50). Quelle est la raison de l'utilisation péjorative par les humains du lexème chien? Si les créatures à deux pattes fuient une araignée, une guêpe et un rat, ont-elles le droit d'affirmer que les chiens s'effraient face aux choses les plus absurdes? Et que dire des oreilles humaines, froides et laides, aux antipodes de celles des canidés, douces et poilues? Voilà la raison qui justifie les boucles, les piercings, voire le culte de l'artificiel. Vers la fin de sa vie, Cross s'attaque à Facebook, son discours accusateur et inquisiteur dénonçant l'aliénation de Mario provoquée par le soi-disant «jouet » intrus et pervers : « Il vaut mieux, Mario, que tu ne laisses pas cette chose-là à ma portée, car je la détruis à coups de dents ${ }^{57}$ » (193).

Toutefois, ce qui intrigue le plus Cross, c'est l'esprit d'imitation ${ }^{58}$ qui débouche sur l'artifice : à Noël, on crée un gros homme, à barbe blanche ${ }^{59}$, qui sent le tissu et le plastique, et on fabrique des arbres artificiels, alors qu'on pourrait en avoir de vrais : "À cette époque de l'année, on ornait des arbres ou des arbustes [...] qui étaient artificiels [...]. De ces arbres-là [...], qui dérangeaient, n'émanait aucune énergie ${ }^{60}$ » (160). Et Cross se hâte de conclure que "C'est en raison de cette chose et de beaucoup d'autres choses que je ne comprends pas l'espèce humanoïde ${ }^{61}{ }$ (160).

Ces quelques divergences sont néanmoins vite résorbées par de significatives affinités comiques, par le biais d'analogies étonnantes, ainsi que par de multiples péripéties vécues par un maître aveugle et son chien guide, à l'image de celles d'un certain Sancho et d'un certain Quichotte. La cravate humaine est ainsi semblable à la laisse, juste un tout petit peu plus large (symbole de dépendance ou soumission d'un homme à l'autre? ou, plutôt, de soumission du chien à l'homme ?) ; les chiens fouillent dans les poubelles, à l'image des hommes qui assaillent les conteneurs (hommes et chiens sans-abri ?); les chiens-guides travaillent (au contraire des mascottes qui se limitent à "être là ", aliénées de la vie) comme les humains, mais gratuitement ou, alors, avec pour seule récompense l'affection (satire sociale débouchant sur l'inégalité des "conditions » et du statut social ?) ; les chiens errants semblent être victimes de l'homme sans cœur, tels les sans-abri humains, éventuels boucs émissaires de la société.

En fait, Cross ne surgit pas, aux côtés du néophyte Mario, comme un chien guide quelconque, mais plutôt comme un "tuteur ${ }^{62}$ "; son rôle, loin d'être celui d'un apprenti, s'avère être celui d'un maître. Dans la dernière lettre que Mario lui adresse, après sa mort, cette inversion de rôles ne peut être plus évidente ; Mario, l'aveugle, non seulement avoue que son chien a changé sa vie, mais reconnaît aussi l'égalité de la race canine et de la race humaine, dans un discours antispéciste :

J'admire et j'essaye d'imiter, dès que cela est possible, votre manière d'agir face à la vie. Pour vous, la rancune, la haine et la tristesse sont fugaces et passent vite. Il y a des gens qui se questionnent sur l'âme des animaux ; quant à moi, je suis d'avis que vous avez une âme, et que votre âme est plus grande, plus pure et plus noble que la nôtre ${ }^{63}$. (235)

Dans ce contexte, la disparition d'un chien ou de son "maître » est un moment foncièrement traumatique pour celui qui subit cette perte. La résignation n'étant pas, dans la majorité des circonstances, l'attitude la plus efficace pour surmonter la douleur, il ne reste (et tout le reste est illusoire) que le recours soit à la «cristallisation» de 
l'image de celui qui a disparu, soit à l'espoir de sa métempsychose, soit à l'éternelle présentification de son absence pénible.

\section{Chiens revenants}

21 Faisant suite à la sentience des chiens, ainsi qu'à la relation particulière qui les unit à l'être humain, il convient d'aborder la thématique de la mort (de l'homme ou du chien) et de la réincarnation (des canidés) dans deux récits cinématographiques et dans une nouvelle.

Dans le film Hachi: A Dogs's Tale (réalisé en 2009 par Lass Hallstrom), inspiré d'une histoire vraie, Hachiko, chien japonais de la race Akita, né en 1923 et décédé en 1935, illustre, d'une façon émouvante, le discours de l'attente obsessive, du désespoir et de l'espoir en simultané : pendant dix ans, il a attendu, patiemment et jusqu'à sa mort, le retour de son maître-ami décédé, faisant preuve d'un discours amoureux tissé de silences et non de mots. Afin d'immortaliser sa fidélité extrême, on lui érigea une statue en bronze sur la Place de la Gare, à Shibuya, puis une autre où il figure en compagnie du professeur disparu ${ }^{64}$.

À son tour, le film A Dog's Purpose (réalisé, également, par Lass Hallstrom en 2017) met en scène le chien Bailey qui, ayant réussi à comprendre au fil du temps quelques mots à ses yeux importants dans la communication humaine, meurt et se réincarne : après avoir vécu plusieurs vies, connu des corps différents, appartenu à différentes races et connu plusieurs avatars, sous le signe du carpe diem ("Vivez le moment présent »), il finit par rencontrer son premier "maître", son meilleur ami dont il fut toujours nostalgique, et qui le reconnaît grâce au langage gestuel, ou mieux, à une plaisanterie qu'il était le seul à pouvoir comprendre.

De même, la nouvelle de l'écrivain et poète portugais Manuel Alegre, intitulée Cão como nós, se révèle emblématique du retour du chien aimé et à tout jamais disparu, moyennant l'alternance des chapitres: les uns, plus longs, sont consacrés au récit de certaines tranches de vie de l'épagneul breton Kurika; les autres, d'une brièveté évocatrice et symbolique (due à son décès), glorifient l'absence devenue présence du canidé, car «les chiens reviennent aussi ${ }^{65}$ » (Alegre 2012: 9). Ce souffle fantastique, à peine atténué par un frein réaliste, connaît maintes occurrences: quand le père du narrateur meurt, Kurika, son chien, se met en boule, comme d'habitude, devant son fauteuil désormais vide; ensuite, et après le décès de Kurika, le narrateur ressent sa présence à ses côtés : d'une part, une "absence assise " (celle de son père disparu), d'autre part une " absence mise en boule » (celle du chien de son père qui est devenu le sien) (70-71). De même, le dialogue-monologue du sujet écrivant est traversé par le vide : « un chien que l'on ne voit pas » (47), « une laisse sans rien » (59) et « un chien que je suis le seul à ressentir » (ibid.). Cette tension angoissante est, enfin, perceptible quand le narrateur s'écrie : « je ne peux entrer dans la salle et voir à nouveau ton cadre $v^{v i d e}{ }^{66} »(33)$. Rebelle, désobéissant et capricieux ${ }^{67}$, Kurika, en proie à l'émotion, est expert dans les rapports avec les humains (69), ainsi que fin connaisseur du sens de son existence : "Quelqu'un a parlé de la tristesse et du vide du regard des animaux. [...] La tristesse de qui veut parler, mais qui ne réussit pas à le faire. Le vide se trouve peut-être dans nos yeux ${ }^{68} »(85)$. D'ailleurs, il n'aboie pas comme les autres chiens : il parle en aboyant, car son rêve est celui de devenir le premier chien à prononcer un mot (31); il parle avec ses yeux, sa queue, son allure, sa tête inclinée; il parle en dressant et en 
baissant ses oreilles (89); il parle, même quand il se tait, à travers les modulations variables de son silence, façonnant une langue à lui, celle d'un canidé humain.

Le poème-épitaphe Cão como nós, que nous traduisons en français, caractérise, d'une façon magistrale, ce chien, qui était un chien, qui ne voulait pas être un chien et qui était un «Chien comme nous ».

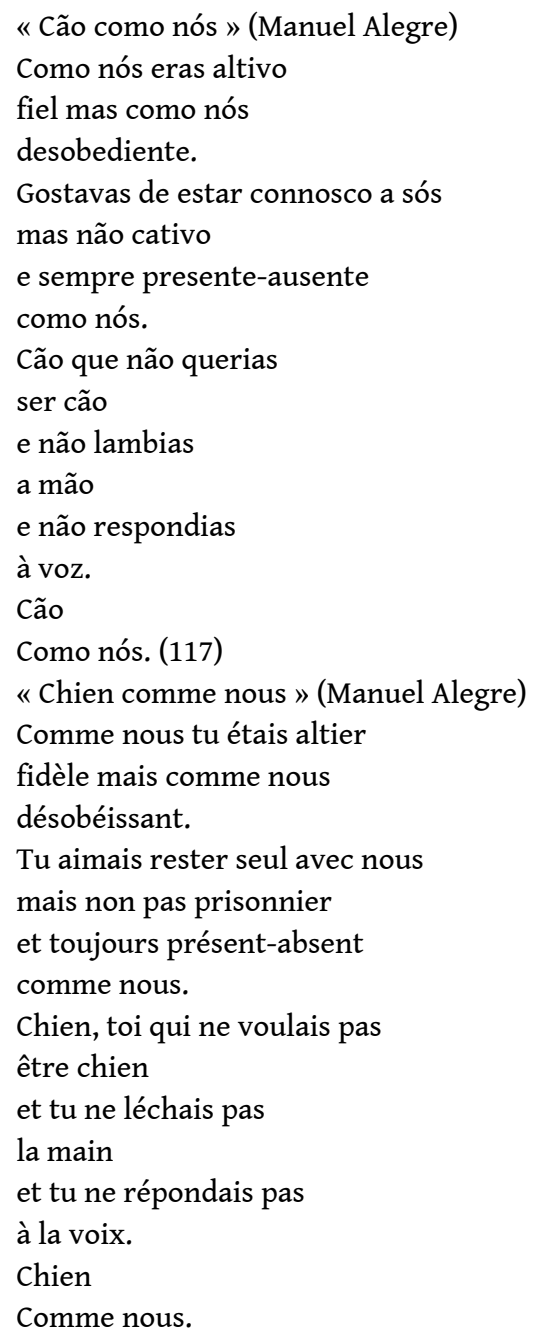

\section{Conclusion}

26 Il est indéniable que, depuis plusieurs années, le paradigme spéciste, anthropocentrique et logocentrique est relativisé au profit de l'antispécisme. Les récentes études scientifiques démontrent que les animaux, et tout particulièrement les chiens, sont des êtres sentients ayant, par conséquent, le droit d'être respectés. Or, ce respect devrait impliquer la transformation de l'homme à grande échelle, le refus de la violence infligée à l'animal et la solidarité envers le vivant.

Ce n'est pas par hasard que les chiens sont considérés comme nos « meilleurs amis ${ }^{69} »$ : en fait, les hommes et les canidés s'apprivoisent mutuellement, grâce à un apprentissage conjoint qui va de pair avec le développement, la formation et l'éducation de l'un et de l'autre. Flush, Mr. Bones et Cross en sont des exemples immortalisés par la littérature : leurs émotions (l'euphorie, l'anxiété, la panique) se mêlent à celles des humains avec lesquels ils partagent l'espace domestique; leur 
discours, quoique différent du langage humain, s'exprime à travers des signes extérieurs (la tête basse, l'agitation de la queue), des sons émis par les organes vocaux (les cris, les aboiements, les hurlements, les gémissements) et l'expression du regard susceptible de traduire la douleur intense, la plainte aiguë et l'euphorie sans limites. Flush et Madame Barrett Browning, Willy et Bonesy, ainsi que Mario et Cross sont des couples indissociables: le premier, chien de compagnie, termine ses jours comme narrateur de ses «tranches de vie " passées à Whitechapel et Wimpole Street, devant un public de chiens italiens ; Bonesy voit son rêve concrétisé, juste avant le moment du suicide : la rencontre avec son ami à Timbuktu ; Cross devient «babysitter » des enfants de Mario, qui grandissent au fur et à mesure que sa fin approche. Quant à Kurika, chien du narrateur de Cão como nós, il est un épagneul breton qui comprend le langage humain, à l'image de Flush, Bonesy et Cross, mais qui ne réussit pas à s'exprimer. D'ailleurs, il est rebelle et désobéissant, il fait des caprices, il se considère et est considéré comme un membre de la famille et, surtout, «il est un chien qui ne voulait pas être chien ", « un chien comme nous ».

Ce qui ressort de la construction de ces couples littéraires, c'est surtout l'unité hommeanimal, au niveau fonctionnel et émotionnel: Flush et Elizabeth, Mr. Bones et Willy ainsi que Cross et Mario sont interdépendants et indissociables. En fait, si les chiens peuplent la solitude des humains, ceux-ci peuplent, sans aucun doute, la leur.

\section{BIBLIOGRAPHIE}

Ariès, Philippe et Duby, Georges (dir.), 1999, Histoire de la vie privée. De la Première Guerre Mondiale à nos jours, Paris, Seuil.

Augé, Marc, 1992, Non-Lieux. Introduction à une anthropologie de la modernité, Paris, Seuil, La Librairie du $\mathrm{XX}^{\mathrm{e}}$ siècle.

Baudelaire, Charles, 1979, Euvres Complètes, Paris, Seuil.

Bentham, Jeremy, 1789, An Introduction to the Principles and Morals and Legislation, Oxford, Clarendon Press.

Broom, Donald M., 2014, Sentience and Animal Welfare, Wallingford, Oxfordshire, CABI.

Broom, Donald M., 2017, Le bien-être animal dans l'Union européenne, Bruxelles, Parlement européen.

Burgos, Jean, 1982, Pour une poétique de l'imaginaire, Paris, Seuil.

Chevalier, Jean et Gheerbrant, Alain, 1969, Dictionnaire des symboles, Paris, Robert Laffont.

Citati, Pietro, 2003, Portraits de femmes, trad. Brigitte Rénol, Paris, Gallimard.

Dallenbach, Lucien, 1977, Le récit spéculaire. Essai sur la mise en abyme, Paris, Seuil.

Damásio, António, 1998, O Erro de Descartes, Mem Martins, Publicações Europa-América.

Damásio, António, 2000a, O Sentimento de Si, Mem Martins, Publicações Europa-América. 
Damásio, António, 2000b, o mistério da consciência. Do corpo e das emoções ao conhecimento de si, São Paulo, Companhia das Letras.

Darwin, Charles, 1890, L'expression des émotions chez l'homme et les animaux, Paris, C. Reinwald.

Deleuze, Gilles et Félix Guattari, 1980, Mille Plateaux, Paris, Les Éditions de Minuit.

Deleuze, Gilles, 2004, Abécédaire (DVD réalisé par P.-A. Boutang, avec G. Deleuze e C. Parnet comme acteurs).

Derrida, Jacques, 2002, 0 animal que logo sou, trad. du français par Fábio Landa, São Paulo, Editora UNESP.

Descartes, René, 1996, CEuvres de Descartes, Discours de la Méthode et Essais, Paris, Librairie Philosophique J. Vrin, vol. VI.

Evrard, Franck, 1999, L’Humour, Paris, Hachette Supérieur.

Fontenay, Élisabeth de, 1998, Le Silence des bêtes : la philosophie à l'épreuve de l'animalité, Paris, Fayard.

Foucault, Michel, [1961] 1972, Histoire de la folie à l'âge classique, Paris, Gallimard.

Grogan, John, 2016, [Marley \& me] Marley e eu, Alfragide, Leya.

Haraway, Donna, 2003, The Companion Species Manifesto: Dogs, People and the Significant Otherness, Chicago, Prickly Paradigm Press.

Highsmith, Patricia, 2002, [Posthumous Short Stories] Contos Póstumos, Mem Martins, Publicações Europa-América.

Kant, Immanuel, 1964, Fundamentação da metafísica dos costumes, São Paulo, Editora Nacional.

Moreira, Alexandra Reis, 2018, "O caso particular dos animais de companhia », dans M. Neves et F. Araújo (dir.), Ética Aplicada. Animais, Lisbonne, Edições 70, p. 183-203.

Neves, Maria do Céu Patrão et Araújo, Fernando (dir.), 2018, Ética Aplicada. Animais, Lisbonne, Edições 70.

Neves, Maria do Céu Patrão et Araújo, Fernando (dir.), 2018, " Bio-diversidade e Co-Existência », Ética Aplicada. Animais, Lisbonne, Edições 70, p. 9-29.

Pelluchon, Corine, 2011, Éléments pour une éthique de la vulnérabilité, Paris, Éditions du Cerf.

Reynier, Christine, 2012, "Virginia face à Victoria », Le Magazine Littéraire, n 518, p. 64-65.

Santos, Ricardo R. et Mestrinho, Lisa, 2018, « Um cão é um cão, um cão, um cão ", dans M. Neves et F. Araújo (dir.), Ética Aplicada. Animais, Lisbonne, Edições 70, p. 205-226.

Simon, Anne, 2011, «Introduction », L’Esprit Créateur, vol. 51, n 4, p. 1-5.

Simon, Anne, 2017a, « Du peuplement animal au naufrage de l'Arche : la littérature entre zoopoétique et zoopoéhique », L'Esprit créateur, vol. 57, nº 1, p. 83-98.

Simon, Anne, 2017b, « Animots/Animaux : Criação literária e Zoopoética [séculos XX e XXI] », dans Cristina Álvares, Ana Lúcia Curado, Isabel Cristina Mateus et Sérgio Guimarães de Sousa (dir.), Figuras do Animal, Braga, Universidade do Minho, Centro de Estudos Humanísticos.

Singer, Peter, [1975] 2015, La Libération animale, Paris, Payot et Rivages.

Thoreau, Henry David, [1854] 2014, Walden ou a vida nos bosques, Lisbonne, Antígona. 
Wilson, Edward, 1999, Consilience: the unity of Knowledge, New York, First Vintage Books Random House.

Wunenburger, Jean-Jacques, 2013, L'Imaginaire, Paris, PUF.

\section{Corpus}

Alegre, Manuel, 2002, Cão como nós, Lisbonne, D. Quixote.

Auster, Paul, [1999] 2008, Timbuktu, Croydon, Faber and Faber.

Hallström, Lass et Cameron, Bruce, 2009, Hachi: A Dog's Tale, États-Unis, 93 minutes.

Hallström, Lass, 2017, A Dog's Purpose, États-Unis, 98 minutes.

Ortiz, Emilio, 2016, A través de mis pequeños ojos, Barcelone, Duomo Ediciones.

Woolf, Virginia, [1933] 2016, Flush. A Biography, Londres, Penguin Little Black Classics.

\section{NOTES}

1. Voir la conception cartésienne de "animal-machine " et de "machines sans âme", dans Discours de la Méthode, « Cinquième Partie » (1996 : 54-60).

2. D'après Kant, les animaux ne sont pas auto-conscients et n'existent que comme instruments ayant une seule finalité : l'Humanité. L'homme est, en fait, le seul être qui a comme attributs la dignité et l'auto-conscience. Voir, à ce propos, Kant, Fundamentação da metafísica dos costumes (1964 : 435-436).

3. Dans L'Origine des Espèces (1859), Darwin développe deux idées fondamentales: l'évolution et l'adaptation des espèces aux circonstances environnantes et la sélection naturelle tendant à favoriser les organismes plus aptes à survivre et à se reproduire. Dans The Descent of Man and Selection in Relation to Sex, le philosophe naturaliste, qui a parcouru, à bord du "Beagle ", l'Amérique du Sud, l'Afrique et l'Océanie pendant cinq ans, affirme que tout ce qui est considéré comme humain (le langage, l'amour maternel et la moralité) a été développé à partir des non-humains.

4. Le terme spécisme, créé par Richard Ryder en 1970 (Experiments on Animals), a été redéfini en 1975 par le psychologue dans un essai intitulé Victims of Science, où il met en rapport le spécisme et le racisme comme étant des préjugés basés sur des apparences.

5. Dans son "Introduction" à L'Esprit Créateur, $\mathrm{n}^{\circ} 4$, Anne Simon se penche sur la différence entre les champs critiques anglo-saxons et français. Relativement au domaine anglo-saxon, l'objet est «moins souvent abordé sous l'angle spécifique des études littéraires que sous celui-ci, plus global, des sciences humaines et sociales ». Par rapport au domaine français, «c'est tout récemment que les études littéraires portant sur des œuvres de langue française intègrent des réflexions jusque-là éparses dans des groupes de recherche $[. .] ».(2011: 2)$.

6. D'après Charles Baudelaire, la modernité est « le transitoire, le fugitif, le contingent, la moitié de l'art dont l'autre moitié est l'éternel et l'immuable » ([1863] 1979 : 553).

7. Selon Marc Augé, la surmodernité est caractérisée par trois figures de l'excès : la surabondance événementielle, la surabondance spatiale et l'individualisation des références (1992: 55). Ce qui distingue, dans la perspective de ce sociologue, la 
surmodernité de la modernité, c'est que, à la différence du "paysage » moderne de Baudelaire, où tout se mêle, où l'on assiste à l'imbrication de l'ancien et du nouveau, la surmodernité fait de l'ancien un spectacle spécifique, consacrant dans ses «non-lieux » une place aux curiosités.

8. Voir, à ce propos, dans Ética Aplicada. Animais (Neves et Araújo 2018), l'article d'Alexandra Reis Moreira intitulé "O caso particular dos animais de companhia" (183-203) / «Le cas particulier des animaux de compagnie » et l'article de Ricardo R. Santos et Lisa Mestrinho intitulé « Um cão é um cão, um cão, um cão » / « Un chien est un chien, un chien, un chien » (205-226).

9. «Sentience » pour les Anglo-Saxons et « Senzienza » pour les Italiens.

10. Voir à ce propos Bentham (1789); voir également Neves et Araújo (2018).

11. Il est évident que le concept polysémique de "sentience» peut être étudié de plusieurs points de vue et dans un large spectre de domaines de spécialité : en biologie (sensibilité et conscience animale), en philosophie (expériences subjectives) et dans le domaine de la spiritualité, l'hindouisme par exemple (respect de la non-violence). Nous avons choisi la perspective antispéciste, qui ne postule aucune hiérarchie entre les espèces et prône la protection animale.

12. En ce qui concerne l'expression des émotions, Darwin (1890) établit trois principes : le principe des habitudes utiles associées, illustré par le cas des chiens qui, avant de se coucher, ont l'habitude de se tourner sur eux-mêmes (dans une tentative d'aplatir l'herbe, comme le faisaient leurs ancêtres) ; le principe de l'antithèse (exemplifié par les intentions amicales des chiens, leur attitude de soumission et d'affection traduite par le fait de remuer la queue ou mordre la main de leur maitre) et le principe des actions dues au système nerveux et indépendantes soit de la volonté soit de l'habitude (dont le paradigme réside dans les signes extérieurs de quelques émotions, comme la douleur intense et la peur, extériorisées par le poil hérissé et le grognement).

13. Pour Deleuze et Guattari (1980), il y a trois sortes d'animaux : les animaux œdipiens qui nous invitent à régresser et nous entraînent dans une contemplation narcissique (les chiens, par exemple), les animaux de genre, comme les grands mythes divins les traitent, et les animaux démoniaques.

14. Par rapport à la mythologie, il convient de mentionner Anubis, la divinité funéraire de l'Égypte antique, représenté comme un homme à tête de canidé et, dans l'Odyssée homérique, Argos, chien d'Ulysse, qui le reconnaît au retour de la guerre de Troie. Les mosaïques de Pompéi témoignent également de la relation ancienne qui unit le chien et l'homme : à l'entrée de quelques maisons («La casa del Poeta Tragico»), figurait un chien avec l'inscription "Cave Canem »; dans ce contexte, il est intéressant de ne pas passer sous silence les ornements de «La Villa dei Misteri » et regarder, dans « La Casa del Fauno ", une scène de chasse à laquelle participent deux chiens. De même, la peinture de la Renaissance, classique, réaliste et impressionniste corroborent la présence récurrente des chiens : les tableaux de Jan Van Eyck (Les époux Arnolfini, 1434), de Claude Gellée dit Le Lorrain (Paysage avec les noces d'Isaac et de Rébecca, 1848), de Diego Velázquez (Les Demoiselles, 1656), de Gustave Courbet (L'Atelier du peintre, 1854), d'Edgar Degas (La classe de danse, 1874), de Pierre-Auguste Renoir (Le déjeuner des canotiers, 1880-1881) et de Georges Seurat (La Grande-Jatte, 1884-1886).

15. L'expression «Le chien est le meilleur ami de l'homme " semble être née lors d'un jugement, dans une petite ville du Missouri, en 1855 : faisant suite à un procès 
concernant l'assassinat d'un chien, l'avocat du parti plaignant a proféré un discours émouvant devant le jury, dans lequel il vantait les multiples qualités du chien, en particulier son amitié inconditionnelle (Santos et Mestrinho $2018: 215$ ).

16. Le 22 janvier 1982, le juge aux affaires matrimoniales du tribunal de grande instance de Meaux rend une ordonnance de non-conciliation, arrêtant que chacun des époux conservera ses objets personnels et que sera remis à l'épouse un chien lui appartenant. L'époux, M.G., demande à bénéficier à l'égard de ce chien d'un « droit de visite et d'hébergement pouvant s'exercer les première et troisième fins de semaine de chaque mois, ainsi que pendant diverses périodes de vacances scolaires ». Débouté, M.G. fait appel (Gazette du Palais, 26 janvier 1983, cité dans Ariès et Duby 1999 : 149).

17. Toutes les traductions, de l'anglais, de l'espagnol et du portugais vers le français, sont de notre main.

18. "Here, then, the biographer must perforce come to a pause " $(2016: 86)$; "But though it would be pleasant for the biographer to infer that Flush's life in late middle age was an orgy of pleasure transcending all description [...] " (88). / « Ici, le biographe doit faire par force une pause» (2016: 86); "Quoiqu'il serait agréable pour le biographe d'en déduire que la vie de Flush, pendant la maturité, avait été une orgie de plaisirs transcendant toute description [...] » (88).

19. « live animal » $(2016: 83)$.

20. « waved and mewed by her side » (ibid.).

21. « repulsive presence » (ibid.).

22. «The baby was set on his back and Flush had to trot about with the baby pulling his ears » (ibid. : 84) / «On a mis le bébé sur lui et Flush a dû trotter d'un côté et de l'autre, tandis que le bébé lui tirait les oreilles » (ibid : 84).

23. "And then, strangely enough, Flush found that he returned the baby's affection " (ibid.) / «Et puis, bizarrement, Flush a découvert qu'il rendait au bébé l'affection qu'il lui portait» (ibid.).

24. « led undoubtedly to much misunderstanding » (ibid. : 23).

25. " peculiar intimacy » (ibid.).

26. Dans la perspective de Wilson, « Many kinds of animals [...] live in a different visual world [...] » (1999: 50) / « Plusieurs espèces d'animaux [...] vivent dans un monde visuel différent $[. .] ».(1999: 50)$.

27. «there was a rattling and a crying, a jingling and a shouting, a cracking of whips and a jangling of bells » (73).

28. «lamented poor, dull, damp, sunless, joyless, expensive, conventional England» (75-76).

29. « the only pure-bred cocker spaniel» (74).

30. « red and virile » (89).

31. L'humour, notion fuyante, légère et fluide, mode de représentation apparemment plus inoffensif que l'ironie, "constitue une sorte de "contre-discours" qui désigne les incohérences de l'existence et du monde et relativise les valeurs affirmées par le discours sérieux, en disant, d'une façon innocente, ce qui est " (Evrard 1996 : 135).

32. Timbuktu est une ville située au centre du Mali, qui, menacée d'être engloutie par les sables du Sahara, a été inscrite, en 1988, par l'UNESCO au Patrimoine mondiale. 
33. Cette expression, que l'on peut traduire par « ami loyal ", provient des aventures de The Lone Ranger, que la télévision a popularisées dans les années 1950.

34. " angel trapped in the flesh of a dog » $(2008: 35)$.

35. «Could anything be more obvious? Just turn around the letters of the word dog, and what did you have?» (36).

36. " was a man with the heart of a dog » (30).

37. «A dog has roughly two hundred and twenty million scent receptors, whereas a man had but five million [...]» (39). Il est intéressant de signaler, au long de l' errance de Willy et de Sir Osso, la référence à la maison où Edgar Allan Poe a vécu, de 1832 à 1835 , et à «Poe-land» (46), qui, du point de vue phonétique, est susceptible de se confondre avec Poland [Pologne].

38. «There was so much to absorb, so much evidence to assimilate, decipher, and make sense of that Willy hardly knew where to begin. The wagging tail as opposed to the tail between the legs. The pricked ears as opposed to the flaccid ears. The rolling on to the back, the running into circles, [...] and a hundred other minute particulars [...] » (37).

39. « learning how to speak a new language » (ibid.).

40. "Where the map of this world ends, that's where the map of Timbuktu begins » (49).

41. « Tim-buk-tu. [...] The blunt combination of vowels and consonants rarely failed to stir him in the deepest parts of his soul [...] » (ibid.). Ces sonorités signifiantes façonnent une image qui constitue la "meilleure formulation possible d'une réalité absente de laquelle elle est inséparable et avec laquelle seulement elle prend sens » (Burgos 1982 : 83).

42. Nous définissons imaginaire comme étant, dans l'optique de Jean Burgos (1982: 88), "l'échange incessant de forces opposées et complémentaires ", susceptible d'organiser un espace qui est à la base de la construction du sens que l'on attribue au réel. À son tour, Jean-Jacques Wunenburger considère que l'imaginaire est « un ensemble de productions, mentales ou matérialisées dans des oeuvres, à base d'images visuelles [...] et langagières [...] formant des ensembles cohérents et dynamiques » $(2013: 10)$.

43. "It was called Timbuktu, and from everything Mr. Bones could gather, it was located in the middle of a desert somewhere, far from New York or Baltimore, far from Poland or any other city they had visited in the course of their travels » (48).

44. «[...] in Timbuktu dogs would be able to speak man's language and converse with him as an equal » (50).

45. "With any luck, he would be with Willy before the day was out » (186).

46. Il est intéressant de constater que, parfois, les auteurs pénètrent ou glissent subrepticement dans leurs livres. Un exemple d'une quasi-métalepse se trouve dans le roman de Paul Auster : " a guy named Anster, Omstair, something like that » (Timbuktu, 2008: 67) ; un autre exemple se trouve dans le roman de John Grogan: «Marley... Grogan... de Churchill » (Marley \& me, $2016: 28$ ).

47. Publié à Barcelone en 2016, la huitième édition apparaît un an plus tard.

48. C'est un ami du protagoniste, Mario, qui lui donne l'idée d'écrire un récit en recourant à un canidé narrateur. La surprise initiale ne tarde pas à céder la place au livre à venir : «-¿Quién se va a creer una historia así, Nico? [...] ¿un perro va a narrar 
mi vida?» (209) ; «-Qui peut croire à une histoire comme celle-ci, Nico ? Un chien va raconter ma vie?».

49. « los perrunos guía nos parecemos en cosas muy concretas a nuestros propios amos, por lo menos en cuestiones sentimentales ».

50. "¿Por qué en vez de recurrir a nosotros no intentan hacer lo que nosotros hacemos? ¿Vivir como nosotros vivimos?»

51. « chupas hechas con animales muertos ».

52. Selon Henry David Thoreau, qui anticipe au XIX ${ }^{e}$ siècle les conséquences néfastes de la Révolution industrielle, la Nature et l'état sauvage s'opposent à la culture et à l'état social. Par conséquent, l'homme doit être une parcelle de la Nature, au lieu de devenir un simple membre de la société. Voir, à ce propos, Randonnée, conférence prononcée le 23 avril 1851 au Concord Lyceum, ainsi que Walden ou la vie dans les bois.

53. Le nom de Thoreau est devenu incontournable lors de l'émergence du mouvement écologique, en 1962, et de la parution du livre Silent Spring de la biologiste américaine Rachel Carson.

54. " rara espécie la de estos seres de dos patas » (31). La critique des « humanoïdes » englobe également la capacité d'assurer (ou de ne pas assurer) un travail: "Rara especie la de estos locos seres de dos patas que son capaces de asegurarnos a nosotros el trabajo y ni siquiera se lo aseguran para ellos » (16-17).

55. «Ellos comían cinco veces al día, nosotros solamente una [...] » (34).

56. «Se metió [Mario] dentro de otra habitación que había en la habitación que los seres de dos patas llaman cuarto de baño [...] 》 (50).

57. «Más te vale, Mario, que no dejes la cosa esa a mi alcance, que de dos bocados me lo cargo $"$.

58. « ¿Por qué esta manía de imitarse a sí mismos?» (160).

59. «Incluso a la puerta de algunos comercios se ponía un señor grueso disfrazado con una barba blanca, un gorro rojo y un traje del mismo color » (159).

60. «En aquella época del año también ponían árboles o arbolitos [...] que no eran de verdad. [...] Esos árboles no [...] desprendían ninguna energía, sólo estorbaban ».

61. "Por ésta y muchas otras cosas no comprendo a la especie humanoide ».

62. Un autre exemple de canidé « tuteur » est Baldur (dans le conte intitulé Le meilleur ami de l'homme de Patricia Highsmith), le chien du Dr Edmund Fenton, qui se conduit d'une façon plus civilisée que la majorité des animaux humains. Si son regard parle et surveille, sa dignité et son intelligence corrigent les "déviations" du médecin (Highsmith [1955] 2002 : 24-38).

63. «Admiro e intento imitar siempre que puedo vuestra manera de actuar ante la vida. Para vosotros no existe el rencor, no existe el odio, ni la tristeza que no sea rápidamente pasajera. Hay gente que se pregunta si los animales tenéis alma ; yo diría que no sólo tenéis alma, diría que vuestra alma es más grande, más pura y más noble que la nuestra ».

64. Récemment, les journaux ont publié la photo de Nala - la chienne du footballeur Emiliano Sala, disparu dans un accident d'avion le 21 janvier 2019 -, qui, après avoir longtemps attendu son retour, s'est refusé à quitter la porte de la chapelle funéraire.

65. « os cães também regressam ». 
66. « não posso entrar na sala e ver outra vez a tua moldura vazia ».

67. À l'image de Kurika, Marley apparaît comme un chien rebelle, mais aussi hyperactif, anormal, mentalement instable. Bien qu'il soit «le pire chien du monde », il est, sans aucun doute, «le meilleur ami de l'homme», en accompagnant les moments les plus heureux et malheureux d'une famille en construction (Grogan, Marley \& Me, 2016).

68. "Alguém falou da tristeza e do vazio do olhar dos animais. [...] A tristeza de quem quer chegar à palavra e não consegue. Mas não vi o vazio. 0 vazio está talvez nos nossos olhos.»

69. Voir l'Abécédaire de Deleuze. Voir, également (d'après Gilles Deleuze et Félix Guattari), que "L'écrivain est un sorcier parce qu'il vit l'animal comme la seule population devant laquelle il est responsable en droit » $(1980: 294)$.

\section{RÉSUMÉS}

Si la sentience renvoie à la conscience, à la capacité d'être affecté par des expériences diverses ou des perceptions multiples de l'univers environnant, à la réaction à un stimulus donné et à des sensations physiques et psychologiques, les chiens, parmi les animaux sentients, occupent une place particulière. En effet, les hommes et les chiens s'apprivoisent mutuellement, grâce à un coapprentissage traversé par le langage émotif et le discours critique. En revisitant le parcours existentiel de trois chiens errants (Flush, Mr. Bones et Cross) et de trois chiens revenants (Kurika, Hachi et Bailey), issus d'un corpus d'œuvres littéraires et cinématographiques, on peut constater le degré de sentience qui les caractérise, la représentativité du narrateur (humain et canin), l'unité fonctionnelle et émotionnelle du protagoniste humain et non humain et l'égalité du chien sentient et de l'homme conscient de sa sentience.

If sentience refers to consciousness, to the ability to be affected by various experiences or multiple perceptions of the surrounding universe, to the reaction to a given stimulus and to physical and psychological sensations, dogs have a special place among sentient animals. In fact, humans and dogs tame each other, through co-learning, through emotional language and critical discourse. By revisiting the existential journey of three stray dogs (Flush, Mr. Bones and Cross) and three returning dogs (Kurika, Hachi and Bailey), coming from a corpus of litterary and cinematographic works, we can see the degree of their sentience, the representativeness of the narrator (human and canine), the functional and emotional unity of the human and non-human protagonist and the equality of a sentient dog in relation to a man conscious of his sentience.

\section{INDEX}

Keywords : sentience, dog, language, emotion, criticism

Mots-clés : sentience, chien, langage, émotion, critique 
AUTEUR

MARIA DO ROSÁRIO GIRÃO RIBEIRO DOS SANTOS

Université du Minho, Portugal 